\title{
Modifizierte Schaumsklerosierung: vielversprechende Ergebnisse
}

Cavezzi A et al. Catheter Foam Sclerotherapy of the Great Saphenous Vein, with Perisaphenous Tumescence Infiltration and Saphenous Irrigation. Eur J Vasc Endovasc Surg. 2017;54:629-635. doi:10.1016/j.ejvs.2017.08.004

Verglichen mit der chirurgischen Intervention und anderen minimal-invasiven Verfahren ist die ultraschallgestützte Schaumsklerosierung (UGFS) mit geringeren Okklusionsraten assoziiert. Dies gilt insbesondere bei größeren Gefäßdurchmessern. Die technische Modifikation von Cavezzi et al. sieht drei Ergänzungen für die UGFS bei einer Varikosis der V. saphena magna (GSV) vor: Die Schaumfreisetzung über einen 4-French-Katheter, die perivenöse Infiltration mit TumeszenzSubstanz und die Spülung mit Kochsalzlösung. Dabei soll der Katheter eine gleichmäßige Schaumverteilung gewährleisten, die Tumeszenz den Venendurchmesser reduzieren und die Spülung eine nahezu vollständige Blutfreiheit vor der Schaumapplikation ermöglichen. Das Verfahren wurde mit einer Phlebektomie variköser Seitenäste kombiniert.
Insgesamt 82 Erkrankte (88 Beine) erhielten die Behandlung nach dem neuen Protokoll. In allen Fällen lag eine primäre Varikosis mit einer Klappeninsuffizienz der GSV (Reflux $>0,5 s$ ) und der terminalen Klappe (SFJ) vor. Der GSV-Durchmesser betrug 7,1 mm (median). In der Mehrzahl bestanden die CEAP-Klassen C2 $(n=43)$ und C3 $(n=21)$. Die Kanülierung erfolgte problemlos. In 3 Fällen ließ der Katheter sich nicht ausreichend vorschieben und stoppte $15-30 \mathrm{~cm}$ vor der SFJ. Durchschnittlich erfolgten 3 Injektionen mit insgesamt 140 ml Tumeszenzlösung und die Spülung mit 40 ml Kochsalzlösung. Die mittlere Dosis des Natrium-Tetradecylsulfat-Schaums betrug $7 \mathrm{ml}$.

5 Patienten nahmen postinterventionell wenige Tage nichtsteroidale Antiphlogistika ein. In den ersten zwei Wochen trat 1 Thrombophlebitis auf. 2 Patienten berich- teten von einer Hautverhärtung und leichten Pigmentierung. 3 Patienten empfanden vorübergehend Parästhesien. Varikosis-assoziierte Beschwerden nahmen postinterventionell signifikant ab. Nach 40 Tagen, 6 Monaten und nach 1 Jahr kamen keine klinischen Rezidive vor. 4 Patienten (4,7\%) hatten nach 3 Jahren sichtbare Rezidivvarizen. Die sonografischen Okklusionsraten betrugen zu den Nachsorgezeitpunkten $100 \%$, $100 \%, 94,3 \%$ und 89,4\%. 9 Patienten hatten nach 36 Monaten einen GSV-Durchmesser von 1,4 mm. 6 Gefäße wiesen einen anterograden Flow auf und in 3 Fällen bestand ein Reflux. Einschränkende Faktoren für die guten Resultate waren laut den Autoren die geringe Patientenzahl, fehlende Vergleichsgruppe und der späte erste Nachsorgetermin.

Dr. med. Susanne Krome, Melle 